Instituto Internacional de Investigación y Desarrollo Tecnológico Educativo INDTEC, C.A.

DOI: https://doi.org/10.29394/scientific.issn.2542-2987.2016.1.2.2.28-41

OAI-PMH: http://www.indteca.com/ojs/index.php/Revista Scientific/oai

\title{
Animación Sociocultural como Estrategia Integradora entre Institución Educativa-Docente y Comunidad
}

\author{
Autora: Ayari del Carmen Gelvis González \\ Universidad Nacional Experimental "Rafael María Baralt", UNERMB \\ ayagel69@hotmail.com \\ Zulia, Venezuela
}

\section{Resumen}

La investigación tuvo como propósito proponer la animación socio cultural como estrategia de integración entre institución-educativa, escuela, docentes y comunidad, en la Escuela Integral Bolivariana El Muro, Municipio Valmore Rodríguez. Estado Zulia. Teóricamente se basa en los autores: Carides (2010), Trillas (2012), Henríquez y Paredes (2015). El estudio se desarrolló mediante la metodología de investigación- acción-participativa, definida como argumento central para la transformación de la problemática, se consideró el modelo Elliot G. (2012), constituido por la acción - reflexión; y organizado por la planificación - observación para una continua interacción que establezca una dinámica conducente a resolver los problemas que tienen lugar en la vida cotidiana de la institución. Para recopilar información, se aplicó la técnica de la observación participante, utilizando grupo focal, el árbol de problemas, lo cual permitió identificar los segmentos de la población más afectadas, lo cuales están interesados en participar en las actividades. Entre los instrumentos utilizados se encuentra la guía de observación, notas de campo, arrojando la carencia en la puesta en práctica de valores, poco sentido de pertenencia, distanciamiento con la comunidad, atribuyendo esto a factores tanto internos como externos, para ello, se hizo necesario la intervención, llegando a extraer conclusiones, recomendaciones, y ofreciendo propuestas hacia la importancia de agitar la animación socio- cultural, como compromisos para impulsar la calidad educativa, mediante estrategias de transformación de los espacios que se conviertan en verdaderos escenarios de integración.

Palabras clave: animación; estratégica; integración; escuela; comunidad.

Fecha de Recepción: 30-09-2016 Fecha de Aceptación: 15-10-2016 


\title{
Sociocultural Animation as Integrating Strategy Between Educational Institution-Teacher and Community
}

\begin{abstract}
This research was aimed at proposing the sociocultural animation as integrating strategy between the educational institution, school, teachers and community, at the Integral Bolivarian School "El Muro" from Valmore Rodríguez municipality in Zulia state. This investigation is theoretically based on the authors: Carides (2010), Trillas (2012), Henriquez and Paredes (2015). The study was developed through the participatory action research methodology, defined as the main argument for the transformation of the problem, the Elliot G. model (2012) was considered, constituted by action-reflection; and organized by planning-observation for a continuous interaction which establishes a dynamic leading to solve the problems that occur in the daily life of the institution. In order to gather information, the participatory observation technique was applied, using focus group and the problems tree, which allowed identifying the most affected parts of the population, which are interested in participating in the activities. Among the instruments used we find the observation guide, field notes, showing the lack of implementation of values, little sense of belonging, distancing from the community, attributing this to both internal and external factors, for this, the intervention was needed, reaching conclusions, recommendations, and offering proposals for the importance of empowering sociocultural animation as commitments to promote quality education through strategies of transformation of spaces that become real integrating scenarios.
\end{abstract}

Keywords: animation; strategy; integration; school; community. 


\section{Introducción}

El proceso curricular venezolano está sustentado en una concepción educativa y pedagógica construida a partir de procesos de integración y participación de las familias, escuela, comunidad, donde la exigencia del Ministerio del Poder Popular para la Educación pide la acción colectiva de todos los responsables del hecho educativo, fundamentada en las potencialidades, habilidades, destrezas proyectadas a través de actividades socioculturales como estrategia para fortalecer el proceso educativo del estudiante.

En este sentido la animación sociocultural es un sistema de dinamización que permite la participación constante de todos los actores del hacho educativo a su vez permite desarrollar capacidades creativas del individuo donde se busque el crecimiento participativo en los procesos socios culturales de una sociedad.

Al respecto, puede señalarse que el currículo Bolivariano completa el empuje a esa denominada animador Sociocultural, con la finalidad de implementar la metodología de estrategia de intervención para promover el desarrollo comunitario a través de la cultura como medio para la creación, imaginación, experiencia colectiva, experimentación de modos de vida con más calidad, la asociación voluntaria, la solidaridad, la autonomía personal para tomar decisiones, el pensamiento reflexivo y el sentimiento de pertenencia a la comunidad

\subsection{Contexto Real de la Situación}

La investigación se realizó en la Escuela Básica Integral El Muro, ubicada en la Carretera Bachaquero -El Venado con Avenida No. 74 del Municipio Valmore Rodríguez del Estado Zulia, su entorno comunitario presenta características especiales considerando que está situado en una vía principal, sector residencial rural, el cual alberga a una población numerosa de 
estudiantes pertenecientes a diferentes estratos sociales, los cuales van desde

grupos en pobreza extrema hasta colectividades con una calidad de vida acorde a las necesidades básicas de cualquier ser humano.

Cabe agregar, la particularidad de la institución educativa la cual se encuentra en el renglón de instituciones Bolivarianas con horario de clases (08:00 am hasta las 04:00 pm), diferente al resto de las escuelas, donde se imparte en las mañanas el contenido curricular dispuesto por el Ministerio del Poder Popular para la Educación, en el horario de la tarde se realizan actividades complementarias tales como: talleres de títeres, danzas, reciclajes, educación deportiva, entre otras.

En este tipo de instituciones los estudiantes reciben una alimentación balanceada, la cual contempla desayuno, almuerzo y merienda, se rigen por el Programa de alimentación Escolar del Gobierno Bolivariano, implementado con la finalidad de mejorar la calidad de vida de los y las estudiantes de entornos con menos poder adquisitivo, es decir, sus familias cuentan con bajos recursos económicos, debido a la carencia de fuentes de empleo formales, la mayoría trabajan en el comercio informal, en casa de familias y en parcelas agrarias.

\subsection{Percepción de la Investigadora}

La organización educativa en estos últimos años busca la figura del animador cultural para implementar estrategias de agitación, promoción y difusión de actividades sociales y culturales que contribuyan a alcanzar la participación e integración de todos los actores educativos, para ello se hace necesario la formación de los miembros, que incentiven la conservación de tradiciones de las comunidades, así como las costumbres autóctonas.

En este sentido, se plantea la Animación Socio-Cultural como un conjunto de técnicas, conocimiento, desde un sistema de dinamización, cuyo objetivo es desarrollar la capacidad creativa del individuo en busca de su 
crecimiento participativo en los procesos socio-culturales de una sociedad, que fortalezca las bases de la construcción de conocimientos, valores, actitudes requeridas para el desarrollo multifacético de los educandos como personas libres, activos, críticos, conscientes para el mejoramiento de su calidad de vida, la de su comunidad y la de toda la sociedad.

De allí que durante el desarrollo de la investigación se viene observando la poca participación e integración desde la comunidad hacia la institución educativa incluyendo los docentes hacia su entorno comunitario, a pesar de tener la figura de los colectivos institucionales existen carencias para aportar propuestas conjuntas en beneficio de todos los miembros del hecho educativo, dejando la mayoría de las veces las soluciones de los problemas de los estudiantes en manos de los docentes de la institución educativa, evidenciando falta de compromiso, poca responsabilidad por fomentar actividades culturales, así como la apatía para planificar este tipo de eventos que permitan rescatar las tradiciones culturales.

Bajo esta percepción la investigadora considera la importancia de analizar la realidad, evaluar las necesidades, diseñar acciones de acuerdo al diagnóstico, ejecutar para mejorar o cambiar la realidad, transformándola para conseguir un cambio real, un verdadero resultado producto de las actividades realizadas, así como reflexionar, evaluando si es necesario volver a actuar hasta lograr transformar esa situación.

Al respecto, Carides (2010), visualiza la animación cultural como una práctica sociocultural educativa relevante para el desarrollo social e individual, actuando como mediadora entre la tradición y el cambio, mediante un amplio conjunto de actores sociales, movimientos asociativos, culturales, administraciones públicas, empresas, organizaciones no gubernamentales, instituciones educativas entre otras, se sientan corresponsables del quehacer cotidiano, fomentando actitudes, comportamientos estimuladores, 
comunicación, participación cívica, creatividad, capacidad expresiva, autorrealización individual y la transformación social.

Para la UNESCO (2011), la animación sociocultural es el conjunto de prácticas sociales cuya finalidad es estimular la iniciativa y la participación de las comunidades en el proceso de su propio desarrollo dentro de la dinámica global de la vida sociopolítica en la cual están integradas. Cabe agregar, que en Sudamérica la Educación Popular, se basa en la idea de que todos los pueblos tienen su propia cultura, toda persona, aunque sea analfabeta, por pertenecer a un determinado territorio cuenta, al menos, con los elementos de su propia cultura. La Educación Popular va dirigida a los sectores más desfavorecidos y pretende por medio de su concientización, un papel activo de los sectores populares en la vida social.

En este sentido, se considera la animación socio cultural como una herramienta que está al alcance de los docentes de las instituciones educativas, la cual, debe ser utilizada para lograr una comunicación efectiva entre los docentes, organización y comunidad a fin de establecer mecanismos de interrelación tales como el rescate de las tradiciones, la participación e integración de todos los actores de la institución educativa con su entorno, constituyendo un avance en la escuela objeto de estudio, dándole un rol más protagónico a las actividades relacionadas con la cultura a fin de unir esfuerzos en beneficio de los involucrados mediante su participación activa.

Por tanto, la investigadora pretende proponer la utilización de la figura del animador cultural dentro de la organización educativa para implementar estrategias que permitan alcanzar la participación e integración de todos los involucrados en la formación educativa de los ciudadanos, para la conservación de las tradiciones de las comunidades para la cual urge la necesidad de jerarquizar las debilidades en el proceso de investigación. 


\subsection{Propósitos General de la Investigación.}

Proponer la animación socio cultural como estrategia de integración entre institución-educativa, escuela, docentes y comunidad del entorno circundante de la Escuela Integral Bolivariana El Muro del Municipio Valmore Rodríguez del Estado Zulia

\section{Fundamentación Teórica}

\subsection{Animación Sociocultural}

Según Macías (2010), la Animación Sociocultural se convierte en una alternativa para promover la participación efectiva, concreta de la comunidad o grupo social en la fijación de prioridades, la toma de decisiones, la elaboración y puesta en marcha de estrategias de planificación para alcanzar un mejor nivel de cultura, la fuerza motriz de este proceso proviene del poder real de las comunidades, de la posesión y del control que tengan sobre sus propios empeños o destinos.

A este respecto, cabe destacar el carácter participativo que le imprime la animación sociocultural para la implementación de estrategias que permitan motivar a los miembros de la comunidad aflorando ese motor interior que posee cada individuo para alcanzar las cosas con su esfuerzo, tesón y las inmensas ganas de construir espacios dignos, seguros, con el esfuerzo de todos los interesados.

Para Trillas (2012), la animación sociocultural es un conjunto de actividades realizadas por grupos, individuos o instituciones sobre una comunidad en el marco de un área geográfica especifica con la finalidad de promover la participación activa de los miembros en el proceso de su propio desarrollo cultural, en este caso específico la escuela pretende utilizar la animación sociocultural como estrategia de intervención para transformar la realidad de la misma. 
Por tanto, el desarrollo de la comunidad se basa en los recursos humanos y materiales con los cuales cuenta la comunidad misma para estimular la independencia, el apoyo social, así como para desarrollar sistemas flexibles que refuercen la participación pública, el control de las cuestiones culturales, esto requiere un total y constante acceso a la información de su tejido social.

Al respecto, puede señalarse la necesidad de darle más empuje a esa figura contemplada en el currículo Bolivariano denominada animador Sociocultural, con la finalidad de implementar la metodología de estrategia de intervención para promover el desarrollo comunitario a través de la cultura como medio para la creación, imaginación, experiencia colectiva, experimentación de modos de vida con más calidad, la asociación voluntaria, la solidaridad, la autonomía personal para tomar decisiones, el pensamiento reflexivo y el sentimiento de pertenencia a la comunidad.

Por eso contribuye a que las personas identifiquen sus posibilidades, así influye en la confianza, capacidad, potencialidad creativa e innovadora del hombre como constructor - consumidor de su propia cultura que lo dirija hacia el desarrollo social, además, promueve nuevas propuestas para el desarrollo de la cultura considerando el papel protagónico de la comunidad no como una asistencia sino como herramienta para su transformación comunitaria.

\subsection{Relación Escuela-docente-comunidad desde la animación sociocultural}

Según Trillas (2010) plantea que la relación escuela-docentecomunidad constituye el apoyo al centro educativo, para poner en marchan la resolución de problemas que atañen a la institución educativa, de igual manera manifiesta que la interacción de estudiantes, familias, consejos comunales, líderes comunitarios, ex-estudiantes, organizaciones populares, sindicatos, iglesias, es importante porque puede derivar una escuela cultural 
educativa, artística, deportiva, científica, recreativa, para el desarrollo de una sociedad.

En este sentido, surge la necesidad de contar con espacios físicos adecuados tanto en la escuela como en la comunidad para presentaciones de danza y teatro, conciertos, exhibición de cine o video, realización de charlas, conferencias y seminarios.

Sobre la base de las ideas expuestas, se plantea una dinámica de investigación participativa, a través de la cual los estudiantes, perciban la realidad de su comunidad, participen en la búsqueda de respuestas a los problemas y demandas.

Al transformar la realidad, ellos mismos van transformándose, anticipando nuevas formas de inducción, aprendizaje, desarrollo y autodesarrollo, se trata de formar parte, de comprometerse contribuyendo a encontrar soluciones a las necesidades comunitarias.

De igual manera, la comunidad está llamada a hacer un aporte esencial conjuntamente con la escuela en el desarrollo de sujetos participativos, democráticos, disciplinados, creativos, convirtiéndose en un vínculo entre escuela-comunidad.

\subsection{Animador sociocultural en el currículo Bolivariano}

El animador sociocultural es un agente social capaz de impulsar actitudes comunitarias, valorar y sentir que actúa sobre la realidad para transformarla.

Desde la perspectiva educativa, el animador es una persona que busca en todo momento la relación personal y la educación en el cambio de actitudes, valores, estimulando para la acción, sacando del aislamiento a las personas. Una de sus funciones, entre otras, es desarrollar e impulsar el proceso de mejora, mediante la donación de su ser, saber, hacer para que la persona sea más valiosa por sí misma y para la sociedad. A su vez, tiene en cuenta los 
intereses, valores, formas de pensar, posibilidades de personas a las que guía y ayuda.

En este sentido, el animador es educador porque en todo momento pretende, con su ser - hacer, que la persona experimente su crecimiento personal, es decir, que el ser humano sea más valioso en sí mismo y para la sociedad; entendiéndose esta acción como algo ejercido desde fuera hacia dentro, como la nutrición o bien sea orientando la estimulación de las potencialidades de las personas. En uno y otro caso, o en la conjugación de ambas personalidades el animador es educador.

Por esto, el animador es aquel provisto de capacidades para mejorar u optimizar a otras personas, de dinamizar a los demás de tal manera que logren personas más humanas, más sensibles a los problemas de sus vecinos, en definitiva, más felices.

Dentro de las funciones del animador sociocultural se encuentran las siguientes:

- Investigación social, cultural que supone entre otras cosas: analizar el entorno social en el cual se pretende actuar, analizar, formular las nuevas expectativas, descubrir y satisfacer las necesidades.

- Estimular - motivar: fomentando las iniciativas, ofrecer igualdad de oportunidades, repartir liderazgo.

- Catalizar proceso de dinamización: establecer prioridades, desarrollar la creatividad, proporcionar los medios para la planificación y promoción de actividades.

- Relación intergrupal: favorecer intercambios de ideas, potenciar la participación.

- Relaciones extra grupo e intergrupal: confiar en la capacidad del grupo, decidir colectivamente, hacer que cada grupo asuma su responsabilidad. 
- Programación: presentar proyectos, priorizar objetivos según las necesidades, e integrar los recursos del medio en la programación.

De igual firma, el Animador Sociocultural debe ser una persona capaz de crear valores en la sociedad en la cual está inmerso, con el fin de que las personas se puedan realizar, dentro de un marco democrático con los derechos, deberes que ello lleva consigo, por ello, admitir que uno de los principales objetivos de la animación sociocultural sea posibilitar al sujeto para constituirse en agente activo de su propia formación, implica admitir a su vez, que el ser humano es perfeccionable a lo largo de su vida porque no existe el pretendido carácter fijo, determinado por la herencia, ni existe el determinismo el cual haría del hombre un mero producto de los factores del medio. En definitiva, el hombre tiene la capacidad de ser perfectible, de ser educable, ello se apoya en las capacidades de aprender, escuchar y ser estimulada que tiene la persona.

\section{Metodología}

Para el desarrollo de este trabajo se seleccionó la investigación-acción apoyada en Elliot (2012); dirigir al trinomio (Escuela- Familia- Comunidad) para que reflexionen sobre su experiencia, destrezas y habilidad educativa.

De igual manera el modelo propuesto está apoyada al autor antes mencionado con el fin de ejecutar acción transformadora en colectivo para mejorar los problemas que afectan a la escuela. Asimismo, la técnica utiliza es la observación y entrevista, que sirve para recabar información sobre detalles importantes para la investigación

Los instrumentos utilizados, fueron la Guía de observación que permitió al investigador describe los datos considerados de gran relevancia para la 
investigación, y a su vez sirvió como ayuda para el desarrollo del marco referencial.

De igual manera la se utilizó la Notas de campo, para el registro cronológico que servirá de guía para el desarrollo de los ciclos de acción de la investigación acción participativa. Tomando como referencia a Martínez (2014),

\section{Resultados de la Categorización}

Las acciones fueron enfocadas hacia la utilización de la animación sociocultural como una estrategia para integrar la escuela, familia, comunidad para ello se realizaran, talleres, charlas, conversatorios, en cual permitió una transformación en los actores educativo donde se logró el impulso de las actividades socioculturales que sirvió como guía para llevar las vivencias a toda la comunidad del sector así como las comunidades cercanas donde se alcanzó un compromisos de sentido de pertenecía hacia esta organización social como lo es la escuela.

\section{Conclusión}

Dejándose guiar por el propósito de la investigación que es Proponer la animación socio cultural como estrategia de integración entre institucióneducativa, escuela, docentes y comunidad del entorno circundante de la Escuela Integral Bolivariana El Muro del Municipio Valmore Rodríguez del Estado Zulia, se puede decir:

Que la animación sociocultural es una práctica educativa que conjuga el derecho de todo ciudadano a la educación integral, al acceso a la cultura con el derecho y el deber de participar activamente en la vida colectiva, en la estructuración, para la transformación de la realidad.

Quiere decir que la animación sociocultural de estar al servicio de una democracia cultural que implica que toda persona tiene vocación de asumir de 
uno u otro modo, una función de animación de su comunidad para contribuir al desarrollo cultural emergente de todos los que la componen

Esto quiere decir que para llevar a cabo la labor de animación sociocultural en las Comunidades se necesita de una estrategia con un enfoque participativo que permita a los miembros de la misma ser actores del proceso de rescate, conservación y difusión del patrimonio cultural

\section{Referencias}

Elliot, G. (2012). Desafíos de la Investigación Cualitativa Catedrática de Pedagogía Social. Universidad Nacional de Educación a Distancia (UNED). Chile.

Pierre, B. (2010). La animación Sociocultural. Universidad de Francia.

Serrano, G. (2011). Animación Sociocultural como valor de patrimonio. Editorial: NARCEA.

Ministerio del Poder Popular para la Educación (2015). Congreso Nacional por la calidad Educativa. Venezuela.

Ministerio del Poder Popular para la Educación (2014). Líneas estratégicas en el marco del proceso curricular venezolano. Venezuela. 


\section{Gelvis González Ayari Del Carmen \\ e-mail: ayagel69@hotmail.com}

Nacida en Ciudad Ojeda estado Zulia, cursó estudio de

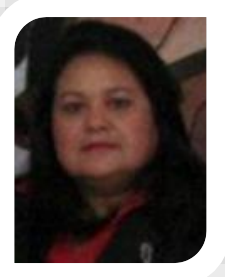
pregrado en la Universidad Nacional Experimental Rafael María Baralt obteniendo título de Lcda. Educación Integral, posteriormente realizó estudios de postgrado en Evaluación por la Universidad Valle del Momboy, estado Trujillo, es Cursante del Doctorado de Educación de la Universidad Nacional Experimental Rafael María Baralt, estado Zulia, Cursante de estudios en Gerencia Educativa de la Universidad Valle de Momboy y Gran Mariscal de Ayacucho, estado Trujillo. Actualmente se desempeña como Subdirectora de la Unidad Educativa Nacional Bolivariana Daniel Mavarez, con 13 años al servicio del Ministerio del Poder Popular para la Educación.

El contenido de este manuscrito se difunde bajo una Licencia de Creative Commons ReconocimientoNoComercial-Compartirlgual 4.0 Internacional 